\title{
Holographic dark energy with varying gravitational constant in Hořava-Lifshitz cosmology*
}

\author{
M. R. Setare ${ }^{1, \dagger}$ and Mubasher Jamil ${ }^{2}$, \\ ${ }^{1}$ Department of Physics, University of Kurdistan, Pasdaran Ave., Sanandaj, Iran \\ ${ }^{2}$ Center for Advanced Mathematics and Physics, \\ National University of Sciences and Technology, Rawalpindi, 46000, Pakistan
}

\begin{abstract}
We investigate the holographic dark energy scenario with a varying gravitational constant in a flat background in the context of Hořava-Lifshitz gravity. We extract the exact differential equation determining the evolution of the dark energy density parameter, which includes $G$ variation term. Also we discuss a cosmological implication of our work by evaluating the dark energy equation of state for low redshifts containing varying $G$ corrections.
\end{abstract}

\section{INTRODUCTION}

Observational data indicates that our universe is currently under accelerating expansion [1 3]. This acceleration implies that if Einstein's theory of gravity is reliable on cosmological scales, then our universe is dominated by a mysterious form of energy. This unknown energy component possesses some strange features, for example it is not clustered on large length scales and its pressure must be negative so that can drive the current acceleration of the universe. Since the fundamental theory of nature that could explain the microscopic physics of DE is unknown at present, phenomenologists take delight in constructing various models based on its macroscopic behavior.

Despite the lack of a quantum theory of gravity, we can still make some attempts to probe the nature of dark energy according to some principles of quantum gravity. An interesting attempt in this direction is the so-called "holographic dark energy" (HDE) proposal [4-7].

\footnotetext{
* M. R. Setare dedicated this paper to the 70 year Jubilee of Professor Farhad Ardalan ${ }^{\dagger}$ Electronic address: rezakord@ipm.ir

${ }^{\ddagger}$ Electronic address: mjamil@camp.nust.edu.pk
} 
The HDE is defined by

$$
\rho_{\Lambda}=\frac{3 c^{2}}{8 \pi G L^{2}}
$$

Here $c^{2}$ is a holographic parameter of order unity and $G$ is a gravitational 'constant'. In this chapter, we shall treat $G$ as a variable. The HDE paradigm has been constructed in the light of holographic principle of quantum gravity [8], and thus it presents some interesting features of an underlying theory of dark energy. Furthermore, it may simultaneously provide a solution to the coincidence problem, i.e why matter and dark energy densities are comparable today although they obey completely different equations of motion [6]. The holographic dark energy model has been extended to include the spatial curvature contribution [9]. Lastly, it has been tested and constrained by various astronomical observations [10].

Recently, a power-counting renormalizable, ultra-violet (UV) complete theory of gravity was proposed by Hořava in [11-14]. Although presenting an infrared (IR) fixed point, namely General Relativity, in the UV the theory possesses a fixed point with an anisotropic, Lifshitz scaling between time and space of the form $\mathbf{x} \rightarrow \ell \mathbf{x}, t \rightarrow \ell^{z} t$, where $\ell, z, \mathbf{x}$ and $t$ are the scaling factor, dynamical critical exponent, spatial coordinates and temporal coordinate, respectively.

Due to these novel features, there has been a large amount of effort in examining and extending the properties of the theory itself [15-27]. Additionally, application of HořavaLifshitz gravity as a cosmological framework gives rise to Hořava-Lifshitz cosmology, which proves to lead to interesting behavior [28, 29]. In particular, one can examine specific solution subclasses [30-32], the perturbation spectrum [33-37], the gravitational wave production [38, 39], the matter bounce [40-42], the black hole properties [43 48], the dark energy phenomenology [49 52], the astrophysical phenomenology [53, 54] etc. However, despite this extended research, there are still many ambiguities if Hořava-Lifshitz gravity is reliable and capable of a successful description of the gravitational background of our world, as well as of the cosmological behavior of the universe [19 21].

In the present work we are interested to study the Holographic dark energy in framework of Hořava-Lifshitz gravity. We extend our analysis with considering the time variable Newton's constant $G$. Until now, in most the investigated dark energy models a constant Newton's "constant" $G$ has been considered. However, there are significant indications that $G$ can by varying, being a function of time or equivalently of the scale factor [55]. In particular, observations of Hulse-Taylor binary pulsar [56, 57], helio-seismological data [58] and astro- 
seismological data from the pulsating white dwarf star G117-B15A [59] lead to $|\dot{G} / G| \lesssim$ $4.10 \times 10^{-11} \mathrm{yr}^{-1}$, for $z \lesssim 3.5[60]$. Additionally, a varying $G$ has some theoretical advantages too, alleviating the dark matter problem [61], the cosmic coincidence problem [62] and the discrepancies in Hubble parameter value [63].

The plan of the paper is as follows: In the second section we shall present a brief overview of Hořava-Lifshitz cosmology. In third section we construct the holographic dark energy with varying gravitational constant and extract the differential equation that determines the evolution of dark energy parameter. In section four, we use these expressions in order to calculate the corrections to the dark energy equation of state for low redshift. Finally in section five, we briefly discuss our results.

\section{HOŘAVA-LIFSHITZ COSMOLOGY}

\section{A. Dark-matter field formulation}

We begin with a brief review of Hořava-Lifshitz gravity. The dynamical variables are the lapse and shift functions, $N$ and $N_{i}$ respectively, and the spatial metric $g_{i j}$ (roman letters indicate spatial indices). In terms of these fields the full metric is

$$
d s^{2}=-N^{2} d t^{2}+g_{i j}\left(d x^{i}+N^{i} d t\right)\left(d x^{j}+N^{j} d t\right)
$$

where indices are raised and lowered using $g_{i j}$. The scaling transformation of the coordinates reads $(z=3)$ :

$$
t \rightarrow l^{3} t \text { and } x^{i} \rightarrow l x^{i}
$$

\section{Detailed Balance}

Decomposing the gravitational action into a kinetic and a potential part as $S_{g}=$ $\int d t d^{3} x \sqrt{g} N\left(\mathcal{L}_{K}+\mathcal{L}_{V}\right)$, and under the assumption of detailed balance [13] (the extension beyond detail balance will be performed later on), which apart form reducing the possible terms in the Lagrangian it allows for a quantum inheritance principle [11] (the $D+1$ dimensional theory acquires the renormalization properties of the D-dimensional one), the full 
action of Hořava-Lifshitz gravity is given by

$$
\begin{aligned}
S_{g}=\int d t d^{3} x \sqrt{g} N\left\{\frac{2}{\kappa^{2}}\left(K_{i j} K^{i j}-\lambda K^{2}\right)-\right. \\
-\frac{\kappa^{2}}{2 w^{4}} C_{i j} C^{i j}+\frac{\kappa^{2} \mu}{2 w^{2}} \frac{\epsilon^{i j k}}{\sqrt{g}} R_{i l} \nabla_{j} R_{k}^{l}-\frac{\kappa^{2} \mu^{2}}{8} R_{i j} R^{i j}+ \\
\left.\quad+\frac{\kappa^{2} \mu^{2}}{8(1-3 \lambda)}\left[\frac{1-4 \lambda}{4} R^{2}+\Lambda R-3 \Lambda^{2}\right]\right\},
\end{aligned}
$$

where

$$
K_{i j}=\frac{1}{2 N}\left(\dot{g_{i j}}-\nabla_{i} N_{j}-\nabla_{j} N_{i}\right)
$$

is the extrinsic curvature and

$$
C^{i j}=\frac{\epsilon^{i j k}}{\sqrt{g}} \nabla_{k}\left(R_{i}^{j}-\frac{1}{4} R \delta_{i}^{j}\right),
$$

is the Cotton tensor, and the covariant derivatives are defined with respect to the spatial metric $g_{i j} . \epsilon^{i j k}$ is the totally antisymmetric unit tensor, $\lambda$ is a dimensionless constant and $\Lambda$ is a negative constant which is related to the cosmological constant in the IR limit. Finally, the variables $\kappa, w$ and $\mu$ are constants with mass dimensions $-1,0$ and 1 , respectively.

In order to add the dark-matter content in a universe governed by Hořava gravity, a scalar field is introduced [28, 29], with action:

$$
\begin{gathered}
S_{m} \equiv S_{\phi}=\int d t d^{3} x \sqrt{g} N\left[\frac{3 \lambda-1}{4} \frac{\dot{\phi}^{2}}{N^{2}}+m_{1} m_{2} \phi \nabla^{2} \phi-\right. \\
\left.-\frac{1}{2} m_{2}^{2} \phi \nabla^{4} \phi+\frac{1}{2} m_{3}^{2} \phi \nabla^{6} \phi-V(\phi)\right]
\end{gathered}
$$

where $V(\phi)$ acts as a potential term and $m_{i}$ are constants. Although one could just follow a hydrodynamical approximation and introduce straightaway the density and pressure of a matter fluid [20], the field approach is more robust, especially if one desires to perform a phase-space analysis.

Now, in order to focus on cosmological frameworks, we impose the so called projectability condition [19] and use an FRW metric,

$$
N=1, \quad g_{i j}=a^{2}(t) \gamma_{i j}, \quad N^{i}=0
$$

with

$$
\gamma_{i j} d x^{i} d x^{j}=\frac{d r^{2}}{1-k r^{2}}+r^{2} d \Omega_{2}^{2}
$$


where $k=-1,0,1$ correspond to open, flat, and closed universe respectively. In addition, we assume that the scalar field is homogenous, i.e $\phi \equiv \phi(t)$. By varying $N$ and $g_{i j}$, we obtain the equations of motion:

$$
\begin{aligned}
H^{2}= & \frac{\kappa^{2}}{6(3 \Lambda-1)}\left[\frac{3 \Lambda-1}{4} \dot{\phi}^{2}+V(\phi)\right]+ \\
+ & \frac{\kappa^{2}}{6(3 \Lambda-1)}\left[-\frac{3 \kappa^{2} \mu^{2} k^{2}}{8(3 \lambda-1) a^{4}}-\frac{3 \kappa^{2} \mu^{2} \Lambda^{2}}{8(3 \lambda-1)}\right]+ \\
+ & \frac{\kappa^{4} \mu^{2} \Lambda k}{8(3 \lambda-1)^{2} a^{2}}, \\
\dot{H}+\frac{3}{2} H^{2} & =-\frac{\kappa^{2}}{4(3 \Lambda-1)}\left[\frac{3 \Lambda-1}{4} \dot{\phi}^{2}-V(\phi)\right]- \\
& -\frac{\kappa^{2}}{4(3 \Lambda-1)}\left[-\frac{\kappa^{2} \mu^{2} k^{2}}{8(3 \lambda-1) a^{4}}+\frac{3 \kappa^{2} \mu^{2} \Lambda^{2}}{8(3 \lambda-1)}\right]+ \\
& +\frac{\kappa^{4} \mu^{2} \Lambda k}{16(3 \lambda-1)^{2} a^{2}},
\end{aligned}
$$

where we have defined the Hubble parameter as $H \equiv \frac{\dot{a}}{a}$. Finally, the equation of motion for the scalar field reads:

$$
\ddot{\phi}+3 H \dot{\phi}+\frac{2}{3 \lambda-1} \frac{d V(\phi)}{d \phi}=0 .
$$

At this stage we can define the energy density and pressure for the scalar field responsible for the matter content of the Hořava-Lifshitz universe:

$$
\begin{aligned}
& \rho_{m} \equiv \rho_{\phi}=\frac{3 \Lambda-1}{4} \dot{\phi}^{2}+V(\phi) \\
& p_{m} \equiv p_{\phi}=\frac{3 \Lambda-1}{4} \dot{\phi}^{2}-V(\phi) .
\end{aligned}
$$

Concerning the dark-energy sector we can define

$$
\begin{aligned}
\rho_{\Lambda} & \equiv-\frac{3 \kappa^{2} \mu^{2} k^{2}}{8(3 \lambda-1) a^{4}}-\frac{3 \kappa^{2} \mu^{2} \Lambda^{2}}{8(3 \lambda-1)} \\
p_{\Lambda} & \equiv-\frac{\kappa^{2} \mu^{2} k^{2}}{8(3 \lambda-1) a^{4}}+\frac{3 \kappa^{2} \mu^{2} \Lambda^{2}}{8(3 \lambda-1)} .
\end{aligned}
$$

The term proportional to $a^{-4}$ is the usual "dark radiation term", present in Hořava-Lifshitz cosmology [28, 29]. Finally, the constant term is just the explicit (negative) cosmological constant. Therefore, in expressions (15), (16) we have defined the energy density and pressure for the effective dark energy, which incorporates the aforementioned contributions. 
Using the above definitions, we can re-write the Friedmann equations (10), (11) in the standard form:

$$
\begin{aligned}
H^{2} & =\frac{\kappa^{2}}{6(3 \lambda-1)}\left[\rho_{m}+\rho_{\Lambda}\right]+\frac{\beta k}{a^{2}} \\
\dot{H}+\frac{3}{2} H^{2} & =-\frac{\kappa^{2}}{4(3 \lambda-1)}\left[p_{m}+p_{\Lambda}\right]+\frac{\beta k}{2 a^{2}} .
\end{aligned}
$$

In these relations we have defined $\kappa^{2}=8 \pi G$ and $\beta \equiv \frac{\kappa^{4} \mu^{2} \Lambda}{8(3 \lambda-1)^{2}}$, which is the coefficient of the curvature term. Additionally, we could also define an effective Newton's constant and an effective light speed [28, 29], but we prefer to keep $\frac{\kappa^{2}}{6(3 \Lambda-1)}$ in the expressions, just to make clear the origin of these terms in Hořava-Lifshitz cosmology. Finally, note that using (12) it is straightforward to see that the aforementioned dark matter and dark energy quantities verify the standard evolution equations:

$$
\begin{aligned}
& \dot{\rho}_{m}+3 H\left(\rho_{m}+p_{m}\right)=0, \\
& \dot{\rho}_{\Lambda}+3 H\left(\rho_{\Lambda}+p_{\Lambda}\right)=0 .
\end{aligned}
$$

\section{HOLOGRAPHIC DARK ENERGY WITH VARYING GRAVITATIONAL CONSTANT IN A FLAT BACKGROUND}

Let us construct holographic dark energy scenario allowing for a varying Newton's constant $G$. The space-time geometry will be a flat Robertson-Walker:

$$
d s^{2}=-d t^{2}+a(t)^{2}\left(d r^{2}+r^{2} d \Omega_{2}^{2}\right)
$$

with $a(t)$ the scale factor and $t$ the comoving time. As usual, the first Friedmann equation reads:

$$
H^{2}=\frac{8 \pi G}{6(3 \lambda-1)} \rho
$$

with $H$ the Hubble parameter, $\rho=\rho_{m}+\rho_{\Lambda}, \rho_{m}=\rho_{m 0} a^{-3}$, where $\rho_{m}$ and $\rho_{\Lambda}$ stand respectively for matter and dark energy densities and the index 0 marks the present value of a quantity. Furthermore, we will use the density parameter $\Omega_{\Lambda} \equiv \frac{8 \pi G}{3 H^{2}} \rho_{\Lambda}$, which, imposing explicitly the holographic nature of dark energy according to relation, becomes

$$
\Omega_{\Lambda}=\frac{c^{2}}{H^{2} L^{2}}
$$


Finally, in the case of a flat universe, the best choice for the definition of $L$ is to identify it with the future event horizon [6], that is $L \equiv R_{h}(a)$ with

$$
R_{h}(a)=a \int_{t}^{\infty} \frac{d t^{\prime}}{a\left(t^{\prime}\right)}=a \int_{a}^{\infty} \frac{d a^{\prime}}{H a^{\prime 2}} .
$$

In the following we will use $\ln a$ as an independent variable. Thus, denoting by dot the time-derivative and by prime the derivative with respect to $\ln a$, for every quantity $F$ we acquire $\dot{F}=F^{\prime} H$. Differentiating (23) using (24), and observing that $\dot{R}_{h}=H R_{h}-1$, we obtain:

$$
\frac{\Omega_{\Lambda}^{\prime}}{\Omega_{\Lambda}^{2}}=\frac{2}{\Omega_{\Lambda}}\left[-1-\frac{\dot{H}}{H^{2}}+\frac{\sqrt{\Omega_{\Lambda}}}{c}\right] .
$$

Until now, the varying behavior of $G$ has not become manifested. However, the next step is to eliminate $\dot{H}$. This can be obtained by differentiating Friedman equation, leading to

$$
\dot{H}=\frac{2 \pi}{3(3 \lambda-1) H}(\dot{G} \rho+G \dot{\rho}),
$$

where $G$ is considered to be a function of $t$. Using

$$
\dot{G}=G^{\prime} H,
$$

and the energy conservation equation

$$
\dot{\rho}=-3 H(1+\omega) \rho,
$$

where $\omega$ is

$$
\omega=\frac{\omega_{\Lambda} \rho_{\Lambda}}{\rho}=\frac{\omega_{\Lambda} \Omega_{\Lambda}}{2(3 \lambda-1)} .
$$

The EoS parameter for HDE is given by

$$
\omega_{\Lambda}=-\left(\frac{1}{3}+\frac{2 \sqrt{\Omega_{\Lambda}}}{3 c}\right)
$$

Eq. (26) becomes

$$
\frac{2 \dot{H}}{H^{2}}=\Delta_{G}-3(1+\omega),
$$

where $\Delta_{G} \equiv G^{\prime} / G$ is a dimensionless number.

$$
\frac{2 \dot{H}}{H^{2}}=\Delta_{G}-3\left[1-\frac{\Omega_{\Lambda}}{2(3 \lambda-1)}\left(\frac{1}{3}+\frac{2 \sqrt{\Omega_{\Lambda}}}{3 c}\right)\right],
$$

Using (32) in (25) we get finally

$$
\frac{\Omega_{\Lambda}^{\prime}}{\Omega_{\Lambda}^{2}}=\frac{2}{\Omega_{\Lambda}}\left[2-\frac{\Delta_{G}}{2}+\frac{\sqrt{\Omega_{\Lambda}}}{2 c}+\frac{\Omega_{\Lambda}}{4(3 \lambda-1)}+\frac{\Omega_{\Lambda}^{3 / 2}}{2 c(3 \lambda-1)}\right]
$$




\section{COSMOLOGICAL IMPLICATIONS}

What we are interested in most is the prediction about the equation of state at the present time. Since we have extracted the expressions for $\Omega_{\Lambda}^{\prime}$, we can calculate $w\left(z^{\prime}\right)$ for small redshifts $z^{\prime}$, performing the standard expansions of the literature. In particular, since $\rho_{\Lambda} \sim a^{-3(1+w)}$ we acquire after expanding $\rho_{\Lambda}$ as

$$
\ln \rho_{\Lambda}=\ln \rho_{\Lambda}^{0}+\frac{d \ln \rho_{\Lambda}}{d \ln a} \ln a+\frac{1}{2} \frac{d^{2} \ln \rho_{\Lambda}}{d(\ln a)^{2}}(\ln a)^{2}+\ldots,
$$

where the derivatives are taken at the present time $a_{0}=1$ (and thus at $\Omega_{\Lambda}=\Omega_{\Lambda}^{0}$ ). Then, $w(\ln a)$ is given as

$$
w(\ln a)=-1-\frac{1}{3}\left[\frac{d \ln \rho_{\Lambda}}{d \ln a}+\frac{1}{2} \frac{d^{2} \ln \rho_{\Lambda}}{d(\ln a)^{2}} \ln a\right],
$$

up to second order. In addition, we can straightforwardly calculate $w\left(z^{\prime}\right)$, replacing $\ln a=$ $-\ln \left(1+z^{\prime}\right) \simeq-z^{\prime}$, which is valid for small redshifts, defining

$$
w(z)=-1-\frac{1}{3}\left(\frac{d \ln \rho_{\Lambda}}{d \ln a}\right)+\frac{1}{6}\left[\frac{d^{2} \ln \rho_{\Lambda}}{d(\ln a)^{2}}\right] z^{\prime} \equiv w_{0}+w_{1} z^{\prime} .
$$

Since $\rho_{\Lambda}=3 H^{2} \Omega_{\Lambda} /(8 \pi G)=\Omega_{\Lambda} \rho_{m} / \Omega_{m}=\rho_{m 0} \Omega_{\Lambda} /\left(1-\Omega_{\Lambda}\right) a^{-3}$, the derivatives are easily computed using the obtained expressions for $\Omega_{\Lambda}^{\prime}$. Hence we get

$$
\begin{aligned}
\omega_{0} & =-\frac{2}{3}-\frac{\Omega_{\Lambda}^{\prime}}{\Omega_{\Lambda}\left(1-\Omega_{\Lambda}\right)} \\
\omega_{1} & =\frac{1}{6}\left[\frac{\Omega_{\Lambda}^{\prime \prime}}{\Omega_{\Lambda}\left(1-\Omega_{\Lambda}\right)}-\frac{\Omega_{\Lambda}^{\prime 2}}{\left(1-\Omega_{\Lambda}\right)^{2}}\left(1+2 \Omega_{\Lambda}+2 \Omega_{\Lambda}^{2}\right)\right] .
\end{aligned}
$$

Using Eq. (33) in (37) and (38), we get

$$
\begin{aligned}
\omega_{0}=- & \frac{2}{3}-\frac{1}{1-\Omega_{\Lambda}}\left[4-\Delta_{G}+\frac{\sqrt{\Omega_{\Lambda}}}{c}+\frac{\Omega_{\Lambda}}{2(3 \lambda-1)}+\frac{\Omega_{\Lambda}^{3 / 2}}{c(3 \lambda-1)}\right] \\
\omega_{1}= & \frac{1}{6}\left[\frac{1}{1-\Omega_{\Lambda}}\left(4-\Delta_{G}+\frac{\sqrt{\Omega_{\Lambda}}}{c}+\frac{\Omega_{\Lambda}}{2(3 \lambda-1)}+\frac{\Omega_{\Lambda}^{3 / 2}}{c(3 \lambda-1)}\right)\right. \\
& \times\left(4-\Delta_{G}+\frac{3 \sqrt{\Omega_{\Lambda}}}{2 c}+\frac{\Omega_{\Lambda}}{3 \lambda-1}+\frac{5 \Omega_{\Lambda}^{3 / 2}}{2 c(3 \lambda-1)}\right) \\
& -\frac{\Omega_{\Lambda}^{2}}{\left(1-\Omega_{\Lambda}\right)^{2}}\left(1+2 \Omega_{\Lambda}+2 \Omega_{\Lambda}^{2}\right) \\
& \left.\times\left(4-\Delta_{G}+\frac{\sqrt{\Omega_{\Lambda}}}{c}+\frac{\Omega_{\Lambda}}{2(3 \lambda-1)}+\frac{\Omega_{\Lambda}^{3 / 2}}{c(3 \lambda-1)}\right)\right]
\end{aligned}
$$


Eqs. (39) and (40) together determine the linear equation of state of dark energy. In order to choose their numerical values and fit them to the observational data, we require a suitable value of $\lambda$. None of the numerical values i.e. $\lambda=1,1 / 3$ or $\infty$ gives a good estimate. Hence we think that the appropriate value of $\lambda$ should be deduced from the observational data.

\section{CONCLUSIONS}

Astrophysical observations suggest that dark energy state parameter must be variable and changing over cosmic time. In this connection, a proposed dark energy candidate is the holographic dark energy. The HDE naturally represents a variable form of dark energy by involving a parameter $c$ in it. The choice $c>1$ leads to the dark energy behaving

as phantom while for $c>1$, in a situation without any other component of energy, the spacetime is not de Sitter. For various reasons, people choose $c=1$.

In this paper, we have analyzed the holographic dark energy with varying gravitational constant in the framework of Hořava gravity. This work is motivated from various astrophysical observations that point out to the notion of variable constants. Although the variations are negligibly small, nevertheless these could be significant in the overall cosmological evolution. It is shown here, the equation of state of dark energy, for lower redshift $z \sim 1$ approximation, is modified due to contribution of varying $G$.

[1] A. G. Riess et al. [Supernova Search Team Collaboration], Astron. J. 116, 1009 (1998);

S. Perlmutter et al. [Supernova Cosmology Project Collaboration], Astrophys. J. 517, 565 (1999).

[2] D. N. Spergel, Astrophys. J. Suppl. 148 (2003) 175;

C. L. Bennett, et al., Astrophys. J. Suppl. 148 (2003) 1;

U. Seljak, A. Slosar, P. McDonald, JCAP 0610 (2006) 014;

D. N. Spergel, et al., Astrophys. J. Suppl. 170 (2007) 377.

[3] E. J. Copeland, M. Sami and Shinji Tsujikawa, Int. J. Mod. Phys. D 15 (2006) 1753, [ arXiv:hep-th/0603057; N. Straumann, arXiv:astro-ph/0009386]; Y. Fujii, Phys. Rev. D 62 
(2000) 064004; L. P. Chimento, A. S. Jakubi and D. Pavon, arXiv:astro-ph/0010079]; J. Kujat, R. J. Scherrer and A. A. Sen, Phys. Rev. D 74 (2006) 083501; Y. -F. Cai, E. N. Saridakis, M. R. Setare, J. -Q. Xia, arXiv:0909.2776 [hep-th].

[4] A. G. Cohen, D. B. Kaplan and A. E. Nelson, Phys. Rev. Lett. 82, 4971 (1999); P. Horava and D. Minic, Phys. Rev. Lett. 85, 1610 (2000); S. D. Thomas, Phys. Rev. Lett. 89, 081301 (2002).

[5] S. D. H. Hsu, Phys. Lett. B 594, 13 (2004).

[6] M. Li, Phys. Lett. B 603, 1 (2004).

[7] K. Enqvist and M. S. Sloth, Phys. Rev. Lett. 93, 221302 (2004); K. Ke and M. Li, Phys. Lett. B 606, 173 (2005); Q. G. Huang and M. Li, JCAP 0503, 001 (2005); B. Wang, Y. Gong and E. Abdalla, Phys. Lett. B 624, 141 (2005); B. Hu and Y. Ling, Phys. Rev. D 73, 123510 (2006); H. Li, Z. K. Guo and Y. Z. Zhang, Int. J. Mod. Phys. D 15, 869 (2006); M. R. Setare, Phys. Lett. B 642, 1 (2006); M. R. Setare, Phys. Lett. B 642, 421 (2006); M. R. Setare, Phys. Lett. B 644, 99 (2007); M. R. Setare, J. Zhang and X. Zhang, JCAP 0703007 (2007); M. R. Setare, Phys. Lett. B 648, 329 (2007); M. R. Setare, Phys. Lett. B 654, 1 (2007); W. Zhao, Phys. Lett. B 655, 97, (2007); M. Li, C. Lin and Y. Wang, JCAP 0805, 023 (2008); M. R. Setare, E. C. Vagenas, Phys. Lett. B 666, 111, (2008).

[8] G. 't Hooft, arXiv:gr-qc/9310026]; L. Susskind, J. Math. Phys. 36, 6377 (1995).

[9] Q. G. Huang and M. Li, JCAP 0408, 013 (2004).

[10] Q. G. Huang and Y. G. Gong, JCAP 0408, 006 (2004); K. Enqvist, S. Hannestad and M. S. Sloth, JCAP 0502004 (2005); J. Shen, B. Wang, E. Abdalla and R. K. Su, Phys. Lett. B 609200 (2005); H. C. Kao, W. L. Lee and F. L. Lin, Phys. Rev. D 71123518 (2005).

[11] P. Horava, arXiv:0811.2217 [hep-th].

[12] P. Horava, JHEP 0903, 020 (2009) [arXiv:0812.4287 [hep-th]].

[13] P. Horava, Phys. Rev. D 79, 084008 (2009) arXiv:0901.3775 [hep-th]].

[14] P. Hořava, arXiv:0902.3657 [hep-th].

[15] G. E. Volovik, arXiv:0904.4113 [gr-qc].

[16] D. Orlando and S. Reffert, arXiv:0905.0301 [hep-th].

[17] T. Nishioka, arXiv:0905.0473 [hep-th].

[18] R. A. Konoplya, arXiv:0905.1523 [hep-th].

[19] C. Charmousis, G. Niz, A. Padilla and P. M. Saffin, arXiv:0905.2579 [hep-th]. 
[20] T. P. Sotiriou, M. Visser and S. Weinfurtner, arXiv:0905.2798 [hep-th].

[21] C. Bogdanos and E. N. Saridakis, arXiv:0907.1636 [hep-th].

[22] J. Kluson, arXiv:0907.3566 [hep-th].

[23] M. Li and Y. Pang, arXiv:0905.2751 [hep-th].

[24] M. Visser, arXiv:0902.0590 [hep-th].

[25] J. Chen and Y. Wang, arXiv:0905.2786 [gr-qc].

[26] B. Chen and Q. G. Huang, arXiv:0904.4565 [hep-th].

[27] F. W. Shu and Y. S. Wu, arXiv:0906.1645 [hep-th].

[28] G. Calcagni, arXiv:0904.0829 [hep-th].

[29] E. Kiritsis and G. Kofinas, arXiv:0904.1334 [hep-th].

[30] H. Lu, J. Mei and C. N. Pope, arXiv:0904.1595 [hep-th].

[31] H. Nastase, arXiv:0904.3604 [hep-th].

[32] M. Minamitsuji, arXiv:0905.3892 [astro-ph.CO].

[33] X. Gao, arXiv:0904.4187 [hep-th].

[34] B. Chen, S. Pi and J. Z. Tang, arXiv:0905.2300 [hep-th].

[35] X. Gao, Y. Wang, R. Brandenberger and A. Riotto, arXiv:0905.3821 [hep-th].

[36] A. Wang and R. Maartens, arXiv:0907.1748 [hep-th].

[37] T. Kobayashi, Y. Urakawa and M. Yamaguchi, arXiv:0908.1005 [astro-ph.CO].

[38] T. Takahashi and J. Soda, arXiv:0904.0554 [hep-th].

[39] S. Koh, arXiv:0907.0850 [hep-th].

[40] R. Brandenberger, arXiv:0904.2835 [hep-th].

[41] R. H. Brandenberger, arXiv:0905.1514 [hep-th].

[42] Y. F. Cai and E. N. Saridakis, arXiv:0906.1789 [hep-th].

[43] U. H. Danielsson and L. Thorlacius, JHEP 0903, 070 (2009) [arXiv:0812.5088 [hep-th]].

[44] A. Kehagias and K. Sfetsos, arXiv:0905.0477 [hep-th];

[45] R. B. Mann, arXiv:0905.1136 [hep-th].

[46] G. Bertoldi, B. A. Burrington and A. Peet, arXiv:0905.3183 [hep-th].

[47] A. Castillo and A. Larranaga, arXiv:0906.4380 [gr-qc].

[48] M. Botta-Cantcheff, N. Grandi and M. Sturla, arXiv:0906.0582 [hep-th].

[49] E. N. Saridakis, arXiv:0905.3532 [hep-th].

[50] A. Wang and Y. Wu, arXiv:0905.4117 [hep-th]. 
[51] C. Appignani, R. Casadio and S. Shankaranarayanan, arXiv:0907.3121 [hep-th].

[52] M. R. Setare, arXiv:0909.0456 [hep-th].

[53] S. S. Kim, T. Kim and Y. Kim, arXiv:0907.3093 [hep-th].

[54] T. Harko, Z. Kovacs and F. S. N. Lobo, arXiv:0908.2874 [gr-qc].

[55] S. D'Innocenti, G. Fiorentini, G. G. Raffelt, B. Ricci and A. Weiss, Astron. Astrophys. 312, 345 (1996); K. Umezu, K. Ichiki and M. Yahiro, Phys. Rev. D 72, 044010 (2005); S. Nesseris and L. Perivolaropoulos, Phys. Rev. D 73, 103511 (2006); J. P. W. Verbiest et al. [arXiv:astro$\mathrm{ph} / 0801.2589]$.

[56] G. S. Bisnovatyi-Kogan, Int. J. Mod. Phys. D 15, 1047 (2006).

[57] Damour T.,et al, Phys. Rev. Lett. 61, 1151 (1988).

[58] D.B. Guenther, Phys. Lett. B 498, 871 (1998).

[59] Biesiada M. and Malec B., Mon. Not. R. Astron. Soc. 350, 644 (2004).

[60] S. Ray and U. Mukhopadhyay, Int. J. Mod. Phys. D 16, 1791 (2007).

[61] I. Goldman, Phys. Lett. B 281, 219 (1992).

[62] M. Jamil, F. Rahaman and M. Kalam, Eur. Phys. J. C 60, 149 (2009). ; M. Jamil et al, Phys. Lett. B 679 (2009) 172

[63] O. Bertolami et al, Phys. Lett. B 311, 27 (1993). 